\title{
A New Thermal-Conscious System-Level Methodology for Energy-Efficient Processor Voltage Selection
}

\author{
Yongpan Liu, Yu Wang, Feng Zhang, Rong Luo, Hui Wang \\ Department of Electronic Engineering, T singhua University, Beijing, 100084, P.R.China \\ ypliu99@mails.tsinghua.edn.cn
}

\begin{abstract}
In this paper, we propose a thermal-conscious system-level methodology to make energy-efficient voltage selection (VS) for namometer processors under real-time constraints. New modeling parameters, such as power composition ratio $(P C R)$, thermal resistance, are integrated and considered in our system models, and their impacts on energy consumption are explored. The interdependence between temperature and power is modeled in an iterative way and two nonlinear programming formulations are presented to determine the optimal energy-efficient supply voltages. Our experiment results show that the energy estimation discrepancy given by the thermal-conscious and traditional system models can reach up to $50 \%$ in $65 \mathrm{~nm} P T M$ CMOS technology, which again underscores the necessity of a thermal-aware power model. Furthermore, our thermalconscious voltage selection (TCVS) approach can achieve up to $12 \%$ further energy savings and much lower peak temperature than the traditional approach. Finally, our specific temperature voltage selection (STVS) approach can reduce the computation complexity greatly with acceptable energy overheads compared with TCVS.
\end{abstract}

\section{INTRODUCTION}

Energy reduction techniques, such as dynamic voltage scaling (DVS), and dynamic power management (DPM) [1-5] are widely adopted in battery-powered digital systems in order to acquire a longer duty life. With the semiconductor manufacturing technology coming into nanometer era, the ratio of leakage power in total power consumption increases steadily. According to Intel's data [6], leakage power contributes about $40 \%$ in its latest high performance microprocessor. Since the processor's operation temperature may vary greatly due to different power profiles of tasks running on it, leakage power also varies greatly because of its exponential relationship with temperature. Therefore, the optimal energy-efficient operation voltage would change according to different leakage power under corresponding processor temperatures, which makes temperature dependent power models a must for energy-efficient voltage selection of each task.

Plenty of research works have been done to deploy DVS techniques for single or multiple DVS-enable processors aiming at energy minimization. [1] proposed a joint approach to combine both DVS and ABB (Adaptive body biasing) techniques so as to reduce both dynamic and leakage power consumption of a low power microprocessor effectively. [2] combined DVS with procrastination to minimize the total energy consumption. [4] used mathematics programming approaches to choose optimal energyefficient supply and body biasing voltage. Given the powercomposition profile of each task, [3] presented a co-synthesis methodology to reduce the system implementation cost of DVS and $\mathrm{ABB}$. However, all above research works neglect the thermal effects on leakage power consumption during task execution, which may lead to great energy and temperature estimation errors in nanometer processors.

Recently, [5] presented a temperature dependent power model at micro-architecture level, and discussed the optimal supply voltage for maximum performance under different cooling conditions. However, neither energy-related issues, such as DVS, nor VS problems under real-time constraints are covered. In this paper, to our best knowledge, we are the first to propose a system-level methodology for optimal VS based on thermal-conscious system model. Our main contributions are listed as below:

I). A novel thermal-conscious system modeling methodology is proposed to evaluate thermal effects on energy consumption and $\mathrm{VS}$, which includes iterative power, thermal and delay models. It is shown that up to $50 \%$ energy consumption is underestimated if thermal effect is omitted.

II). New modeling parameters, such as $P C R$ and thermal resistance are integrated and considered in our system models, and their impacts on energy consumption and VS are also explored.

III). Based on our thermal-conscious system models, two voltage selection procedures: TCVS and STVS are proposed, which are both formulated as nonlinear programming problem. According to our experiment results, TCVS could lead to up to $12 \%$ further energy savings and lower peak temperature compared with traditional VS method. Meanwhile, STVS reduces computation complexity greatly with acceptable energy overheads compared with TCVS.

This paper is organized as below: the motivation of this work is given out in section 2 ; the formulation of voltage selection problem and system-level models are then illustrated in section 3 . Section 4 gives out two energy-efficient voltage selection procedures. Finally, experiment results and future work are reported in section 5 .

\section{Motivation}

Previous VS research works [1-4] didn't consider the operational temperature for tasks, thus they omitted the thermal effects on task's energy estimation and optimization. It may lead to quite large energy estimation deviations in future nanometer processors, where leakage power contributes a substantial part of total power budget. As we can see from [10], the power profile of different tasks for Intel's latest Montecito processor has a very large power variation, from $68 \mathrm{~W}$ to $126 \mathrm{~W}$. Assuming a typical air cooling condition [9], Fig.1 gives out the temperature curve of the processor executing a task set, which contains different tasks with random power profile ranges from 10 to $130 \mathrm{~W}$. It is shown that the processor's temperature varies in a range from 60 to $110^{\circ} \mathrm{C}$. Such big temperature variations actually lead to quite different leakage

Supported by National NSF of China (No. 90207001, No. 60506010) and

National 863 project of China (No. 2005AA1Z1230) 
power consumption and accordingly affect the energy-efficient VS. Therefore, thermal-conscious model must be used in order to acquire accurate energy estimation and VS.

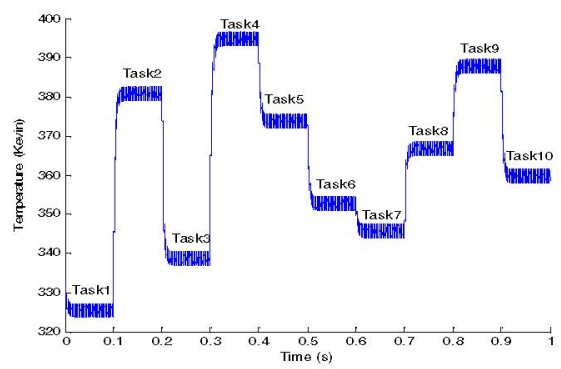

Fig.1 Thermal profiles of running a task set

In traditional VS problem, each task is assumed to be operated at the same temperature under different supply voltages, thus the interdependence between power and temperature is omitted. Based on iterative models, which accurately describe one task's power and thermal profiles, Fig.2 has illustrated the thermal-conscious power profile of one task at different supply voltages. As we can see, the cross and square curves give out the upper and lower bounds of traditional leakage power trends assuming constant temperatures, respectively. The dot curve gives out the accurate iterative leakage power profile. There are a big difference between the traditional trend and the iterative one.

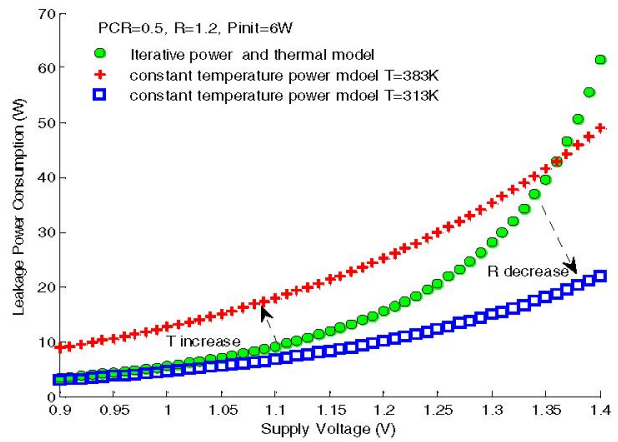

Fig. 2 Thermal-conscious leakage power vs supply voltage

In this paper, a thermal-conscious system model is proposed to revisit the traditional VS problem, and thermal impacts on it will be thoroughly examined. As [8] has shown, typical tasks' power spectrum mainly focuses on a fixed DC component, which can be considered to keep constant during its execution. Therefore, it is firstly assumed that each task's operational temperature only varies within a small margin during its execution, thus we can use the average temperature value to calculate each task's energy consumption without sacrificing its accuracy. Secondly, each task's execution time is assumed at least two magnitudes of thermal constant, which locates in the microsecond magnitude [9], so the processor reach its steady temperature most of the execution time. We may satisfy this by choosing appropriate task granularity.

\section{Preliminaries}

This section presents a thermal-conscious system-level methodology for energy and temperature analysis, and contains the following subsections: VS formulation, power and delay model, thermal model and iterative modeling method.

\section{A. Voltage Selection Formulation}

The energy-efficient VS problem is to find out an optimal operational voltage for each task assigned to certain processor so as to minimize the total energy of multiprocessor system, which is shown in Fig.3. Here, a key difference from traditional approaches is that thermal-conscious parameters, such as $T, P C R, R$ (refer to the following section), are integrated in system models.

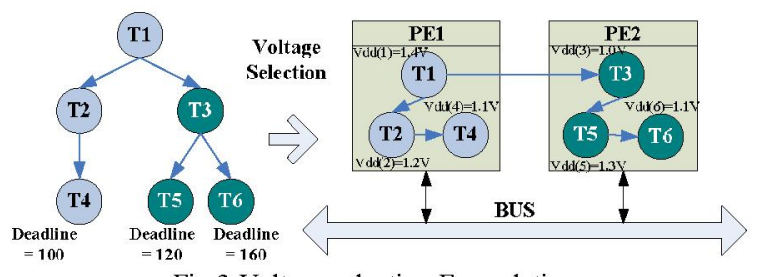

Fig.3 Voltage selection Formulation

The task graph is denoted as $G=(V, E)$, which is a directed acyclic graph composed of a set of $N$ vertices $V=\left\{v_{1}, v_{2} \ldots v_{\mathrm{N}}\right\}$, with each vertex representing a specific task of the graph $G$, and a set $E$ of edges where each edge is denoted by an ordered pair of tasks $(v, v)$, which represents the precedence constraint between these two tasks. For each task $v$, four energy related parameters are defined: the number of execution cycles $E C$, the average switching probability $\alpha^{j}$, the operational temperature $T^{j}$, and the power composition ratio $\left.P C R\right|_{T=T Y Y=V_{\text {min }},}$ which is defined as the ratio between leakage and total power at minimal voltage and ambient temperature for a task. Furthermore, there is a deadline deadline $e^{i}$ associated with each leaf node $v_{i}$ in the task graph $G$, which specifies the real-time constraints. For each processor $P_{\text {; }}$, there are also five technology parameters, namely total switch capacitance, peak running frequency, thermal resistance, maximal and minimal operating voltage, denoted as $C_{\text {efr }}^{i} f_{\max }, R_{i}, V_{\max }$ and $V_{\text {wix }}$, respectively.

\section{B. Power and Delay Model}

Next, we will derive the temperature dependent power model for DVS-enabled processors. The dynamic power consumption of a CMOS microprocessor is temperature insensitive [1], thus it can be acquired by the following formula:

$$
P_{d y n}=C_{e f f} \cdot V_{d d}^{2} \cdot f^{i}
$$

where $C_{\text {eff }}$ is the effective switch capacitance, $f$ is the processor running frequency. Due to our simulation reports [11], the leakage power model is based on the following formula:

$$
P_{\text {leakage }}=V_{d d} \cdot I_{s}\left(T_{0}, V_{d d}^{\min }\right) \cdot\left(A \cdot T^{2} e^{\left(\alpha \cdot V_{d d}+\beta \cdot V_{b s}+\gamma\right) / T}+B \cdot e^{\mu \cdot V_{d d}}\right)
$$

, where $I_{s}$ is the average leakage current under ambient temperature and minimum voltage, $T$ is the operational temperature of the processor, which will be discussed in the next section and other parameters are empirical constants for different circuit types, technologies and designs. All of the technology constants are acquired by using the curve-fitting method. In our experiments [11], 65nm PTM models are adopted to derive those constants. The delay of VLSI circuits is decided by the following formula [1]:

$$
f^{-1}=t=\frac{L_{d} K_{6} V_{d d}}{\left(V_{d d}-V_{t h}\right)^{\sigma}}=\frac{L_{d} K_{6} V_{d d}}{\left(\left(1+K_{1}\right) \cdot V_{d d}+K_{2} \cdot V_{b s}-V_{t h 1}\right)^{\sigma}}
$$




\section{Thermal Model}

According to the heat transfer theory, thermal phenomena can be modeled as an equivalent RC thermal circuit. The $R$ and $C$ values for a specific cooling configuration are derived using Hotspot [9]. The ambient temperature of the processor is denoted as $T_{A}$, and $T_{i m i t}$ is the initial temperature of the processor when executing the $i$ th task: task $k_{i}$ we derive the following formula to calculate the operational temperature $T_{\mathrm{i}}$ :

$$
T_{i}=T_{A}+\left(T_{i n i t}-T_{A}\right) \cdot e^{\frac{-t}{R C}}+P_{i} \cdot R \cdot\left(1-e^{\frac{-t}{R C}}\right)
$$

where $P_{i}$ is the average power consumption of task $k_{i}$. As section 2 has illustrated, assume that $t>>R C$, the following formula can be derived:

$$
T_{i}=T_{A}+P_{i} \cdot R
$$

Since the temperature distribution in a processor is variable, the temperature in the formula should be an average one. It will introduce $(<5 \%)$ leakage power estimation error due to detail simulation results [11].

\section{Iterative Modeling}

Given initial dynamic and static power consumption, each processor converges to a steady temperature under its specific thermal condition. Since operational temperature for each task varies greatly, traditional approach leads to quite large estimation error, which may generate suboptimal VS solution. In this paper, we propose an accurate iterative approach to calculate the steady power consumption and temperature. Since it is encapsulated in TCVS procedure, it will be explained in the next section.

\section{Thermal-Conscious Voltage Selection}

In this section, we will give out two VS formulations based on the thermal-conscious system models.

\section{A. TCVS}

The energy-efficient voltage selection problem under real-time constraints is formulated as the following expression.

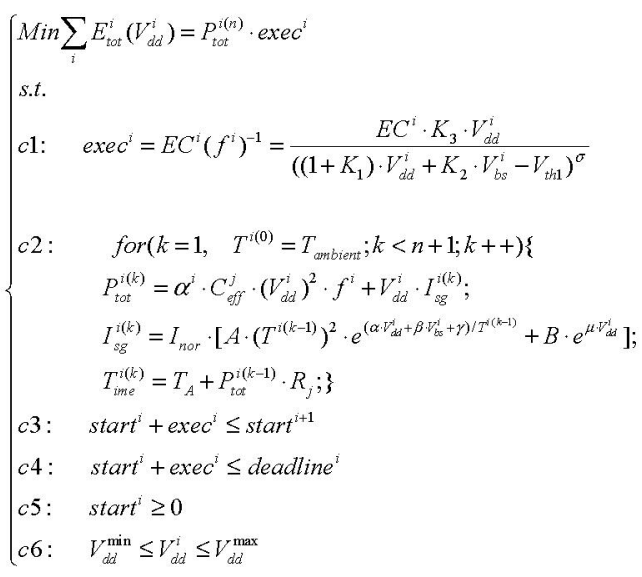

The optimization variables for this problem are the task execution times $\operatorname{exec}^{i}$, the task start times start $^{i}$, and the operational voltages $V_{d d}^{i}$. The goal of this problem is to minimize the total execution energy of all tasks. For each task, constraint $c l$ is based on the delay model, which gives out the relationship between its execution time and supply voltage. Constraint $c 2$ describes the iterative relationship between power and temperature, where $I_{\text {nor }}$ is a real number for each task to make the ratio between dynamic power and leakage power equal to $P C R$ at minimal voltage $V_{\text {min }}$ and ambient temperature $T_{a m b i e n t}, R_{j}$ is the thermal resistance of the assigned processor, and $n$ is a user-specific integer number. The processor's steady power and temperature when executing task will be given out after $n$th iterations. In our experiments, $n$ is usually less than 5 and only $0.01 \%$ errors are observed. Constraint c3 gives out the precedent relationships among tasks in the task set. Since the processor can only execute one task at the same time, resource constraints between two tasks assigned to the same processor are also considered in $c 3$. Constraint $c 4$ satisfies the leaf nodes' deadline. Constraint $c 5$ and $c 6$ are the bounds of variables.

\section{B. STVS}

Next, we propose another formulation to the VS problem. The total VS problem is divided into two phases. In the first phase, a constant temperature is used to estimate the power consumption for each task, and then voltage selection for each task is done. In the second phase, the assigned supply voltage for each task is used as the input for iterative models to calculate its accurate energy consumption. Compared with TCVS, this approach uses the energy under a user-specific constant temperature, thus it may acquire a suboptimal voltage schedule. However, according to our experiment results, energy overheads are usually acceptable. Since the iterative energy and temperature estimation are removed from the nonlinear programming optimization procedure, it greatly reduces the NLP problem's computation complexity.

\section{Experimetn Results And Analysis}

To demonstrate thermal effects on the energy estimation and voltage selections, several experiments are used, and they are solved on a $1.4 \mathrm{Ghz}$ Centrino ${ }^{\mathrm{TM}}$ laptop running Linux. For the largest case, the run time does not exceed $300 \mathrm{~s}$.

The first experiment is to show thermal impacts on VS under different parameters. Supply voltage must be decided for two tasks running on two processors. Parameters for task $k_{1}$ are set as: power $=40 \mathrm{~W}, P C R=0.5$, and it is assigned to processor $P_{1}$ with $R$

$=1.2$; task $_{2}$ has two variable parameters: namely power, PCR, and it is assigned to processor $P_{2}$ with variable thermal resistance; task $_{2}$ 's execution can't start until the completion of task $k_{1}$. Fig.4 gives out the total energy consumption trends under different parameters using different VS methods. Since there are three variables, namely power, $P C R$ and $R$, only one is changeable in each diagram. The energy estimation using non-thermal-conscious (ENT) approach gives out the energy consumption by traditional approach. Since it omits thermal impacts on the energy consumption, it always gives out lower estimation. The curve here is mainly to show how large energy discrepancy between thermalconscious and traditional model. As we can see, up to $50 \%$ energy estimation errors are observed, so it further underscores the necessity of thermal-conscious power modeling method. The STVS curve is drawn by doing voltage selection using a constant user-specific temperature for all tasks, thus its voltage schedule may be suboptimal. However, since its energy consumption would be recalculated by iterative thermal-conscious models, its energy estimation is accurate.

The most energy efficient VS results are given by TCVS curve because of its accurate thermal-conscious energy model. As we can see from Fig.4, TCVS is always better than STVS under all 
different cases. In Fig. $4 \mathrm{~b}$ and Fig. $4 \mathrm{c}$, when the $P C R$ (or $R$ ) difference between task ${ }_{2}$ and task is maximal, the largest energy savings can be acquired. Since only $P C R$ (or $R$ ) is different between task $_{1}$ and task ${ }_{2}$, in STVS both tasks are considered equally, while in TCVS, the task with large $P C R$ (or $R$ ) has a higher priority to be scaled down, because of sharper energy increase with higher voltage. Therefore, the maximum difference in $P C R$ (or $R$ ) leads to up to $12 \%$ energy savings. However, for the power parameter $P$, both STVS and TCVS set high priority to the task with higher initial power consumption, thus the energy difference is trivial

Table.1 Energy Consumption under Different VS Approaches
\begin{tabular}{|c|c|c|c|c|c|}
\hline & $\begin{array}{c}\text { Nodes } \\
/ \text { Edges/PE }\end{array}$ & $\begin{array}{c}\text { STVS } \\
\text { Tmax }\end{array}$ & $\begin{array}{c}\text { TCVS } \\
\text { Tmax }\end{array}$ & $\begin{array}{c}\text { ENT } \\
\text { Errors }\end{array}$ & $\begin{array}{c}\text { Further } \\
\text { Savings }\end{array}$ \\
\hline TS1 & $13 / 12 / 2$ & $245099 / 362$ & $243655 / 365$ & $20.5 \%$ & $0.6 \%$ \\
\hline TS2 & $16 / 15 / 2$ & $280488 / 414$ & $279389 / 398$ & $13.3 \%$ & $0.4 \%$ \\
\hline TS3 & $37 / 40 / 2$ & $757749 / 456$ & $747530 / 397$ & $16 \%$ & $1.3 \%$ \\
\hline TS4 & $66 / 81 / 2$ & $1534056 / 380$ & $1518576 / 380$ & $15 \%$ & $1.0 \%$ \\
\hline
\end{tabular}

In order to illustrate the thermal effects in more general cases, we further testify our VS procedure on random generated benchmark set so as to show its effectiveness, the experiment results are listed in Tab.1. The task graph is generated by TGFF [12]. The $P C R$ and $R$ values of all tasks' are automatically generated random numbers with a uniform distribution, and they locate in the range of $[0.05,0.5]$ and $[0.9,1.0]$, respectively.

As we can see, the energy estimation error between ENT and TCVS is up to $20.5 \%$. Furthermore, TCVS always gets further energy savings compared with STVS, but STVS provides a feasible VS solution with very small energy overheads. Since the $P C R$ and $R$ difference in the random generated benchmarks is not big enough, it makes the energy savings from TCVS not so large as those in the two-tasks case. In addition, TCVS approach can generate selection with lower peak temperature. We can see these in the cases of TS2 and TS3. It is due to the fact that TCVS adopts a thermal-conscious model for VS, which presents a sharp energy increase with higher temperature above certain inflexion. Therefore, TCVS assigns a lower voltage to that task to reduce the energy and accordingly acquires a lower operation temperature. While in STVS, the feedback of energy and temperature is not considered in VS, and the scheduler may assign equal supply voltage to tasks with same power but different $P C R$ and $R$. Thus, processors become quite hot in some cases. Another interesting phenomenon is that though the maximum temperature of the processor varies greatly according to different VS solution, their energy consumption is quite close. This reveals that a thermalconscious optimization voltage selection with small energy consumption overhead can be achieved. Since temperature has an exponential impact on the system reliability [7], our TCVS is quite useful because it not only reduces the energy consumption, but also improves the reliability of nanometer processors.

\section{CONCLUSIONS}

In this paper, we presented a thermal-conscious system-level methodology for VS problem. Iterative methods are used to accurately estimate task energy consumption and processor temperature. New modeling parameters, such as power composition ratio and thermal resistance are integrated and considered in our system models, and their impacts on energy consumption are explored. Based on the thermal-conscious model, energy-efficient voltage selection problem are solved by two nonlinear programming approaches: TCVS and STVS. Compared with traditional VS approaches, our methods can achieve more accurate energy estimation and further energy savings.

\section{REFERENCES}

[1] Martin, M., etc: Combined dynamic voltage sealing and adaptive body biasing for lower power microprocessors under dynamic workloads. ICCAD2002, p 721-725.

[2] Jejurikar, R; Pereira, C; Gupta, R.: Leakage aware dynamic voltage scaling for realtime embedded systems. DAC, June, 2004, p 275-280.

[3] Wu, D. etc: Power-composition profile driven co-synthesis with power management selection for dynamic and leakage energy reduction. DSD, 2005, p 34-40.

[4] Andrei, A Schmitz M. Eles, P. Peng Z - Al-Hashimi, B.M : Overhead-conscious voltage selection for dynamic and leakage energy reduction of time-constrained systems. IEE Proceedings:, v 152, n 1, January, 2005, p 28-38.

[5] Liao, W.P.; He, L.; Lepak, K.M.: Temperature and supply voltage aware performance and power modeling at microarchitecture level, IEEE Transactions on CAD, $v 24, \mathbf{n} 7$, July 2005, p $1042-53$

[6] Http://www.intel.com

[7] Http://public.itrs.net/ Common/2005ITRS/Home2005.htm.

[8] Li, H.; Liu, P.; Qi, Z.Y.; Jin, L.L.; Wu, W.; etc: Efficient thermal simulation for runtime temperature tracking and management, ICCD2005, p 130-133.

[9] Skadron, K.; Stan, M. R; Huang W., etc.: Temperature-aware microarchitecture Conference Proceedings, ISCA, 2003, p 2-13.

[10] Naffziger, S.etc: The implementation of a 2-core, multi-threaded itanium family processor. JSSC, vol. 41, no. 1, Jan. 2006, p197-209.

[11] Zhang, F., Power modeling for microprecessors, BS thesis. Tsinghua Univ. 2006

[12] Dick, R, etc., TGFF: task graphs for free, Proc. CODES, pages 97-101, 1998.

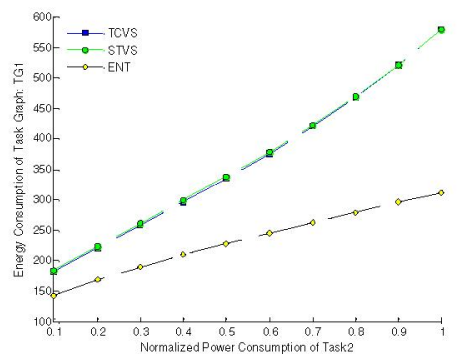

Fig.4.a Energy vs power of $\operatorname{tas}_{2}$

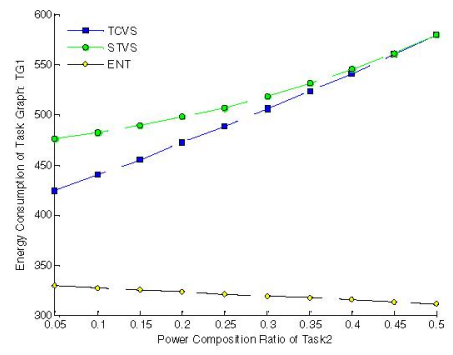

Fig.4.b Energy vs PCR of task

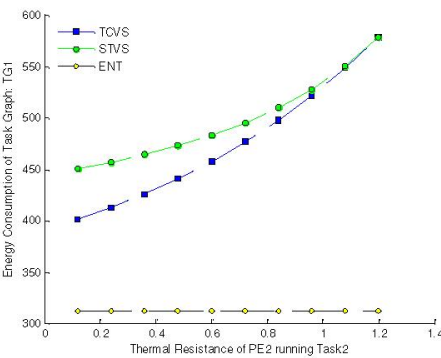

Fig.4.c Energy vs R of task $_{2}$ 